\title{
A New Form of the Spherical Expansion of Zonal Functions and Fourier Transforms of $\mathrm{SO}(d)$-Finite Functions
}

\author{
Agata BEZUBIK ${ }^{\dagger}$ and Aleksander STRASBURGER ${ }^{\ddagger}$ \\ $\dagger$ Institute of Mathematics, University of Biatystok, Akademicka 2, 15-267 Biatystok, Poland \\ E-mail: agatab@math.uwb.edu.pl \\ $\ddagger$ Department of Econometrics and Informatics, Warsaw Agricultural University, \\ Nowoursynowska 166, 02-787 Warszawa, Poland \\ E-mail: strasburger@alpha.sggw.waw.pl
}

Received November 30, 2005, in final form February 17, 2006; Published online March 03, 2006

Original article is available at http://www.emis.de/journals/SIGMA/2006/Paper033/

\begin{abstract}
This paper presents recent results obtained by the authors (partly in collaboration with A. Dąbrowska) concerning expansions of zonal functions on Euclidean spheres into spherical harmonics and some applications of such expansions for problems involving Fourier transforms of functions with rotational symmetry. The method used to derive the expansion formula is based entirely on differential methods and completely avoids the use of various integral identities commonly used in this context. Some new identities for the Fourier transform are derived and as a byproduct seemingly new recurrence relations for the classical Bessel functions are obtained.
\end{abstract}

Key words: spherical harmonics; zonal harmonic polynomials; Fourier-Laplace expansions; special orthogonal group; Bessel functions; Fourier transform; Bochner identity

2000 Mathematics Subject Classification: 33C55; 42B10; 33C80; 44A15; 44A20

This paper is dedicated to Professor Jacques Faraut on the occasion of his 65-th anniversary.

\section{Introduction and preliminaries on spherical harmonics}

Many parts of analysis on Euclidean spaces and in particular the theory of spherical harmonics provide an elegant and instructive application of group theoretical concepts to various questions of classical function theory. It suffices to mention points like: coincidence of eigenspaces of spherical Laplacian with irreducible components with respect to the natural action of the group $\mathbf{S O}(d)$ within the $L^{2}$ space on the sphere; the role of classical orthogonal polynomials, i.e. Gegenbauer polynomials, as reproducing kernels for the spaces of spherical harmonics of a given degree, or more generally, as providing an explicit construction of symmetry adapted basis functions for those spaces (cf. [12]), or the connection of the Fourier transform on the Euclidean space to the Hankel transform obtained via restriction to $\mathbf{S O}(d)$-finite functions and various integral identities of the Hecke-Bochner type resulting there from.

The goal of the present paper is to present under one cover recent developments within this circle of ideas, obtained in $[11,2,3]$ by the present authors, partly in collaboration with A. Dąbrowska. The central point of this paper is a novel form of spherical expansion of zonal functions on Euclidean spheres which we derive by purely differential methods. We show how it implies certain general formulae for the Fourier transform of $\mathbf{S O}(d)$-finite functions, including 
recent generalizations of the famous Bochner formula and also derive certain function theoretic consequences.

\subsection{Preliminaries and notations}

We denote by $(x \mid y)$ the standard (Euclidean) inner product of points $x, y \in \mathbb{R}^{d}$ and by $r=$ $|x|=(x \mid x)^{1 / 2}$ the corresponding length (or radius) function. The unit sphere in $\mathbb{R}^{d}$ is denoted by $S^{d-1}$ and for $x \neq 0$ we shall often write $x=r \xi$ with $\xi \in S^{d-1}$. We shall assume $d \geq 3$ and frequently use a constant $\alpha$ defined by $\alpha=(d-2) / 2$. The sphere is regarded as a homogeneous space of the group $K=\mathbf{S O}(d)$ of (proper) rotations in $\mathbb{R}^{d}$ and for a point $\eta \in S^{d-1}$ its isotropy group $K_{\eta} \subset K$ can be identified with the group $\mathbf{S O}(d-1)$.

Let $\Delta=\sum_{j=1}^{d} \partial^{2} / \partial x_{j}^{2}$ denote the Laplacian in $\mathbb{R}^{d}$. We let $\mathcal{P}^{l}=\mathcal{P}^{l}\left(\mathbb{R}^{d}\right)$ denote the space consisting of homogeneous polynomials of degree $l$ on $\mathbb{R}^{d}$ and denote the kernel of $\Delta$ in $\mathcal{P}^{l}$ by $\mathcal{H}^{l}$. This latter space consists of harmonic and homogeneous polynomials of degree $l$ and its dimension is given by

$$
\operatorname{dim} \mathcal{H}^{l}=\frac{2(l+\alpha) \Gamma(2 \alpha+l)}{\Gamma(l+1) \Gamma(2 \alpha+1)}
$$

where $\Gamma(z)$ denotes the Euler gamma function. Both these spaces are invariant under the natural action of the group $K=\mathbf{S O}(d)$ on functions on $\mathbb{R}^{d}$ given by $k \cdot f(x)=f\left(k^{-1} x\right)$ for $k \in K$, $x \in \mathbb{R}^{d}$. It is known that this group acts irreducibly in $\mathcal{H}^{l}$ for each $l$, and the representations so obtained are inequivalent for $l \neq l^{\prime}$.

The surface spherical harmonics of order $l$ are by definition the restrictions of elements from $\mathcal{H}^{l}$ to the unit sphere $S^{d-1}$, and since the restriction map commutes with the action of rotations the spaces of surface spherical harmonics of any fixed order are invariant and irreducible under $K$.

We endow $\mathcal{P}^{l}=\mathcal{P}^{l}\left(\mathbb{R}^{d}\right)$ with an inner product defined by the formula

$$
\langle P \mid Q\rangle:=\int_{S^{d-1}} P(\xi) \overline{Q(\xi)} d \sigma(\xi)
$$

where $d \sigma(\cdot)$ is the Euclidean surface measure on the unit sphere $S^{d-1}$ normalized so that the total area of the sphere is 1 . In fact, the integral on the right hand side extends to the natural inner product on $L^{2}\left(S^{d-1}, d \sigma\right)$ and it is known that the spaces of spherical surface harmonics of different orders are orthogonal to each other with respect to this inner product.

We recall that a function $f$, defined on the unit sphere $S^{d-1}$, is said to be a zonal function (relative to a point $\eta \in S^{d-1}$ ) if it is invariant with respect to the isotropy group $K_{\eta}$ of $\eta$. Any such function depends in fact on the inner product $(\xi \mid \eta)$ only, and so there exists a function $\phi$ defined on the closed unit interval $[-1,1] \subset \mathbb{R}$ so that

$$
f(\xi)=\phi((\xi \mid \eta)), \quad \text { for all } \xi \in S^{d-1} .
$$

We shall call $\phi$ the profile function of $f$.

\subsection{The canonical decomposition of homogeneous polynomials}

It is well known that every homogeneous polynomial of degree $l$ can be represented as a sum of products of harmonic homogeneous polynomials with even powers of the radius, cf. equation (3) below, and this decomposition is usually called the canonical decomposition of homogeneous polynomials. While the general form of the canonical decomposition, i.e. equation (3), is stated 
in all sources concerned with this matter, the explicit formula for harmonic components of a given homogeneous polynomial is seldom quoted. The only source known to us, where the formula can be found, is the paper [7] of Lucquiaud. We record it here since it is essential for considerations to follow, in particular, in establishing formula (6) giving expansion of elementary zonal polynomials of the form $(x \mid \eta)^{l}$, where $\eta \in S^{d-1}$ is an arbitrary unit vector.

Theorem 1 (The canonical decomposition). The space $\mathcal{P}^{l}$ decomposes orthogonally as the sum $\mathcal{P}^{l}=\oplus_{k=0}^{[l / 2]} r^{2 k} \mathcal{H}^{l-2 k}$ and the decomposition is invariant with respect to the action of the group $\mathbf{S O}(d)$. Explicitly, every polynomial $P \in \mathcal{P}^{l}$ may be written as

$$
P=\sum_{k=0}^{[l / 2]} r^{2 k} h_{l-2 k}(P), \quad \text { where } \quad h_{l-2 k}(P) \in \mathcal{H}^{l-2 k}
$$

are called the harmonic components of $P$ and are given by

$$
h_{l-2 k}(P)=\sum_{j=0}^{[l / 2]-k} e_{j}^{l}(k) r^{2 j} \Delta^{k+j} P
$$

with the coefficients $e_{j}^{l}(k)$ determined by

$$
e_{j}^{l}(k)=(-1)^{j} \frac{(\alpha+l-2 k) \Gamma(\alpha+l-2 k-j)}{4^{k+j} k ! j ! \Gamma(\alpha+l+1-k)} .
$$

The maps $P \mapsto r^{2 k} h_{l-2 k}(P) \in P^{l}$ are projections onto $\mathbf{S O}(d)$-irreducible subspaces of $\mathcal{P}^{l}$ commuting with the action of the group $\mathbf{S O}(d)$.

\section{Expansions of zonal functions}

\subsection{Elementary zonal functions and reproducing kernels}

To proceed, we need to recall an explicit formula for the Gegenbauer polynomial $C_{l}^{\alpha}$ of degree $l$ and index $\alpha$, given for example in [8, Section 5.3.2] or [13, Chapter 9.2], which reads as follows:

$$
C_{l}^{\alpha}(t)=\sum_{j=0}^{[l / 2]}(-1)^{j} \frac{\Gamma(\alpha+l-j)}{\Gamma(\alpha) \Gamma(j+1) \Gamma(l+1-2 j)}(2 t)^{l-2 j} .
$$

Applying the formulae for the canonical decomposition given in (4) and (5) to elementary zonal polynomials $(x \mid \eta)^{l}$, where $\eta \in S^{d-1}$ and $l$ is a nonnegative integer, we obtain

$$
(x \mid \eta)^{l}=2^{-l} \Gamma(\alpha) \Gamma(l+1)|x|^{l} \sum_{k=0}^{[l / 2]} \frac{(\alpha+l-2 k)}{k ! \Gamma(\alpha+l+1-k)} C_{l-2 k}^{\alpha}((\xi \mid \eta)), \quad x=|x| \xi .
$$

In particular, the spherical harmonic obtained by restricting to the unit sphere the harmonic component $h_{l}\left(P_{\eta}\right)$ of the highest degree of $P_{\eta}(x)=(x \mid \eta)^{l}$, which is given by the formula

$$
h_{l}\left(P_{\eta}\right)(\xi)=2^{-l} \Gamma(\alpha) \Gamma(l+1) \frac{(\alpha+l)}{\Gamma(\alpha+l+1)} C_{l}^{\alpha}((\xi \mid \eta)),
$$

plays an important role in the group representation theoretic interpretation of the decomposition (1). With normalization given by

$$
Z_{\eta}^{l}(\xi)=\left[C_{l}^{\alpha}(1)\right]^{-1} C_{l}^{\alpha}((\xi \mid \eta))
$$


it satisfies

$$
\operatorname{dim} \mathcal{H}^{l} \int_{S^{d-1}} Z_{\eta}^{l}(\xi) P(\xi) d \sigma(\xi)=P(\eta)
$$

Because of this property, $Z_{\eta}^{l}(\xi)$ is called the reproducing kernel for the space $\mathcal{H}^{l}$. Moreover, it is uniquely (up to a scalar multiple) determined by the property of being invariant under the action of the isotropy subgroup $K_{\eta}$ of the point $\eta \in S^{d-1}$.

\subsection{A differential formula for expansions of smooth zonal functions}

Now recall $[9,12]$ that every square integrable function on the sphere can be written as a series of spherical harmonics (the Fourier-Laplace expansion). For zonal functions this expansion, thanks to an easy group representation theoretic argument, reduces to

$$
f(\xi)=\sum_{m=0}^{\infty} f_{m} Z_{\eta}^{m}(\xi), \quad \text { where } \quad f_{m}=\operatorname{dim} \mathcal{H}^{m} \int_{S^{d-1}} f(\xi) Z_{\eta}^{m}(\xi) d \sigma(\xi) .
$$

By taking into account the invariance under $K_{\eta}$ of the integrand and equation (2), the coefficients may be expressed as integrals

$$
f_{m}=\frac{(\alpha+m) \Gamma(\alpha)}{\sqrt{\pi} \Gamma(\alpha+1 / 2)} \int_{-1}^{1} \phi(t) C_{m}^{\alpha}(t)\left(1-t^{2}\right)^{\alpha-1 / 2} d t,
$$

what reduces the problem of spherical expansion of zonal functions to the expansion of profile functions $\phi$ with respect to the (orthogonal) system of Gegenbauer polynomials. The following result shows that the coefficients $f_{m}$ of the expansion can also be expressed in terms of the coefficients of the Taylor expansion of the profile function $\phi$, provided the latter satisfies suitable regularity assumptions.

Theorem 2. Assume $\phi:[-1,1] \rightarrow \mathbb{C}$ has the Taylor expansion $\phi(t)=\sum_{m=0}^{\infty} \frac{\phi^{(m)}(0)}{m !} t^{m}$ that is absolutely convergent on the closed interval $[-1,1]$, and let $f(\xi)=\phi((\xi \mid \eta))$ be the zonal function on the sphere $S^{d-1}$ corresponding to $\phi$. Then the spherical Fourier expansion of $f(\xi)$ is given by

$$
f(\xi)=\Gamma(\alpha+1) \sum_{m=0}^{\infty} f_{m} \operatorname{dim} \mathcal{H}^{m} Z_{\eta}^{m}(\xi)
$$

where the coefficients of the expansion can be expressed as

$$
f_{m}=\sum_{k=0}^{\infty} \frac{\phi^{(m+2 k)}(0)}{2^{m+2 k} k ! \Gamma(\alpha+m+k+1)} .
$$

A detailed proof of this result is contained in the forthcoming paper [3] of the authors, and here we shall present its main line only. It consists in substituting the expansion formula (6) into the Taylor series of $\phi$ and rearanging terms so that to group together the terms corresponding to the Gegenbauer polynomials of a given degree. This procedure requires some estimates on the coefficients which assure the absolute convergence of the double series, which are presented in detail in [3].

Below we briefly present two applications of this expansion for obtaining new derivations of some classical results. 


\subsection{The plane wave expansion}

A well known and very useful instance of the expansion (7) is the so called plane wave expansion, giving a representation of the exponential function $e^{i(x \mid \eta)}$ as a series of zonal harmonic polynomials. The expansion involves the Bessel functions of the first kind of order $\nu \in \mathbb{C}$ with $\operatorname{Re} \nu>-1$, which are given as

$$
J_{\nu}(t)=\left(\frac{t}{2}\right)^{\nu} \sum_{k=0}^{\infty} \frac{(-1)^{k}}{\Gamma(k+1) \Gamma(k+\nu+1)}\left(\frac{t}{2}\right)^{2 k}, \quad t \in \mathbb{C}
$$

For some purposes the results are better expressed with the aid of the so called spherical Bessel functions, which are given as

$$
j_{\nu}(t)=\Gamma(\nu+1)\left(\frac{t}{2}\right)^{-\nu} J_{\nu}(t)
$$

We point out that all classical proofs of this formula known to us are obtained by applying certain integral identities of the Hecke-Bochner type, as in [5, 9]. With the use of (7) we get it by direct differentiation of the exponential $e^{i r t}$ and comparison with the power series expansion of the Bessel function (8).

Corollary 1. For an arbitrary unit vector $\eta \in S^{d-1} \subset \mathbb{R}^{d}$ and $x \in \mathbb{R}^{d}$, the plane wave $e^{i(x \mid \eta)}$ admits the following expansion

$$
e^{i(x \mid \eta)}=\sum_{m=0}^{\infty} i^{m} \operatorname{dim} \mathcal{H}^{m} \frac{\Gamma(\alpha+1)}{\Gamma(\alpha+m+1)}\left(\frac{r}{2}\right)^{m} j_{\alpha+m}(r) Z_{\eta}^{m}(\xi), \quad x=r \xi, \quad \xi \in S^{d-1}
$$

The series converges absolutely on each sphere of radius $r$ and uniformly with respect to $\xi, \eta \in$ $S^{d-1}$.

\subsection{The generating function of Gegenbauer polynomials}

Take a fixed element $x \neq 0$ from the unit ball in $\mathbb{R}^{d}$ and write $x=r \eta, \eta \in S^{d-1}$. The function

$$
S^{d-1} \ni \xi \mapsto|\xi-x|^{-2 \alpha}=\left(1-2 r(\xi \mid \eta)+r^{2}\right)^{-\alpha}
$$

is clearly a zonal function with pole at $\eta$ corresponding to the profile function $\left(1-2 r t+r^{2}\right)^{-\alpha}$. The expansion resulting from (7) has the form

$$
\left(1-2 r(\xi \mid \eta)+r^{2}\right)^{-\alpha}=\sum_{m=0}^{\infty} \frac{\Gamma(\alpha+m)}{\Gamma(2 \alpha) \Gamma(m+1)} r^{m} Z_{\xi}^{m}(\eta)
$$

and can be reduced to the familiar formula for the generating function of Gegenbauer polynomials

$$
\left(1-2 r t+r^{2}\right)^{-\alpha}=\sum_{m=0}^{\infty} r^{m} C_{m}^{\alpha}(t)
$$

as given e.g. in $[1,5,8]$. 


\section{The Fourier transforms of $\mathrm{SO}(d)$-finite functions}

In this section we shall present some applications of the plane wave expansion given in Corollary 1 to problems of Fourier analysis in Euclidean space. The subject is related to the well known Bochner formula (c.f. e.g. [1,9]), which describes the restriction of the Fourier transform to the space of functions of the form $f(r) P(x)$, where $f$ is a radial function and $P \in \mathcal{H}^{l}$ a homogeneous harmonic polynomial. The result is again a product of the same harmonic polynomial $P$ with a radial function, which is expressed as the so called Hankel transform of the original radial factor and is given by the formula (12) below. The result we present in Corollary 4 generalizes that relation for the case of arbitrary homogeneous polynomials and is a combination of results obtained by the authors in [11, 2] and by F.J. Gonzalez-Vieli in [6].

\subsection{The case of $\mathrm{SO}(d)$-finite functions on the sphere}

We take the Fourier transform of suitable regular (e.g. $L^{1}\left(\mathbb{R}^{d}\right)$ ) functions on $\mathbb{R}^{d}$ as defined by

$$
\mathcal{F} f(x)=(2 \pi)^{-\frac{d}{2}} \int_{\mathbb{R}^{d}} e^{i(x \mid y)} f(y) d y, \quad x \in \mathbb{R}^{d} .
$$

Observe that the above definition of the Fourier transform also makes sense in the case when $f$ is a function defined on the unit sphere - in this case it can be regarded as the Fourier transform of a measure supported on the sphere. Especially interesting is the case, when the measure on the sphere comes from restricting a homogeneous polynomial to the sphere, since this way we obtain a so-called $\mathbf{S O}(d)$-finite measure (since its $\mathbf{S O}(d)$-translates span a finite dimensional subspace).

Theorem 3. If $P \in \mathcal{P}^{l}$, then the Fourier transform of the measure $P(\xi) d \sigma(\xi)$ with support on the unit sphere $S^{d-1}$ is given by the following equivalent formulae

$$
\begin{aligned}
\mathcal{F}(P) & =\int_{S^{d-1}} e^{i(x \mid \eta)} P(\eta) d \sigma(\eta) \\
& =\left(\frac{i}{2}\right)^{l} \sum_{k=0}^{[l / 2]} \frac{(-1)^{k} 2^{2 k} \Gamma(\alpha+1)}{\Gamma(\alpha+l+1-2 k)} j_{\alpha+l-2 k}(|x|) h_{l-2 k}(P)(x) \\
& =\left(\frac{i}{2}\right)^{l} \sum_{k=0}^{[l / 2]} \frac{(-1)^{k} \Gamma(\alpha+1)}{k ! \Gamma(\alpha+l+1-k)} j_{\alpha+l-k}(|x|)\left(\Delta^{k} P\right)(x)
\end{aligned}
$$

with $h_{l-2 k}(P)$ denoting the harmonic components of $P$ as in equation (3).

The formula (10) has been derived by F.J. Gonzalez [6] and the equivalence of those two forms was observed by the present authors and A. Dąbrowska in [2].

Theorem 3 implies the following interesting function theoretical corollary. By comparing the two expressions (9) and (10) from this theorem one may derive the following multi-step recurrence relation for spherical Bessel functions.

Corollary 2. If $\alpha \geq 0$ is a half odd integer (or an integer) then for any $l \in \mathbb{Z}_{+}$the following relations hold

$$
j_{\alpha+l-s}(r)=\sum_{k=0}^{s} \frac{s ! \Gamma(\alpha+l+1-s) \Gamma(\alpha+l-k-s)}{k !(s-k) ! \Gamma(\alpha+l+1-k) \Gamma(\alpha+l-2 k)}\left(\frac{r}{2}\right)^{2(s-k)} j_{\alpha+l-2 k}(r)
$$

for $s=1, \ldots,[l / 2]$. 
In terms of the Bessel functions of the first kind, this is expressed by

$$
\frac{1}{s !}\left(\frac{2}{r}\right)^{s} J_{\alpha+l-s}(r)=\sum_{k=0}^{s} \frac{\Gamma(\alpha+l-k-s) \Gamma(\alpha+l+1-2 k)}{k !(s-k) ! \Gamma(\alpha+l+1-k) \Gamma(\alpha+l-2 k)} J_{\alpha+l-2 k}(r) .
$$

It is interesting to note that these latter relations unify several classical relations satisfied by Bessel functions like [1, equation (4.6.11)] or [8, Chapter 3.2.2]. In fact, taking into account the relation

$$
J_{-1 / 2}(t)=\left(\frac{1}{2} \pi t\right)^{-1 / 2} \cos t
$$

which follows directly from the expansion (8), and the familiar recurrence relations satisfied by $J_{\nu}(t)$, namely

$$
\left(\frac{1}{t} \frac{d}{d t}\right)^{l}\left(t^{-\nu} J_{\nu}(t)\right)=(-1)^{l} t^{-\nu-l} J_{\nu+l}(t)
$$

one can derive from (11) the following relations.

Corollary 3 (Finite expansions of Bessel functions). The Bessel functions of integer order satisfy the relation

$$
\frac{1}{n !}\left(\frac{2}{t}\right)^{n} J_{n}(t)=\sum_{k=0}^{n} \epsilon_{k} \frac{1}{(n+k) !(n-k) !} J_{2 k}(t), \quad \text { where } \quad \epsilon_{k}= \begin{cases}1, & k=0 \\ 2, & k=1,2,3 \ldots,\end{cases}
$$

while those of half odd integer order satisfy

$$
\begin{aligned}
J_{n+1 / 2}(t)= & \sqrt{\frac{2}{\pi x}}\left\{\sin (t-n \pi / 2) \sum_{k=0}^{[n / 2]} \frac{(-1)^{k}(n+2 k) !}{(2 k) !(n-2 k) !(2 t)^{2 k}}\right. \\
& \left.+\cos (t-n \pi / 2) \sum_{k=0}^{[(n-1) / 2]} \frac{(-1)^{k}(n+2 k+1) !}{(2 k+1) !(n-2 k-1) !(2 t)^{2 k+1}}\right\} .
\end{aligned}
$$

\subsection{The generalized Bochner formula and the Hankel transform}

For our last topic recall that for suitable functions on the nonnegative real axis $\mathbb{R}_{+}$, say, with $\phi$ belonging to the Schwartz space $\mathcal{S}(\mathbb{R})$, one defines the Hankel transform by the formula

$$
H_{\nu}(\phi)(t)=\frac{2^{-\nu}}{\Gamma(\nu+1)} \int_{0}^{\infty} \phi(s) j_{\nu}(s t) s^{2 \nu+1} d s .
$$

Theorem 3 above now immediately implies that the generalized Bochner formula which was previously obtained in [11] can also be expressed by a pair of equivalent formulae.

Corollary 4 (Generalized Bochner identity). If $P \in \mathcal{P}^{l}$, then the Fourier transform of the function $f(|x|) P(x)$ is given by the following expressions:

$$
\begin{aligned}
(2 \pi)^{-\frac{d}{2}} \int_{\mathbb{R}^{d}} f(|x|) P(x) e^{i(y \mid x)} d x= & i^{l} \sum_{k=0}^{[l / 2]}(-1)^{k} H_{\alpha+l-2 k}\left(s^{2 k} f\right)(|y|) h_{l-2 k}(P)(y) \\
& =i^{l} \sum_{k=0}^{[l / 2]} \frac{(-1)^{k}}{2^{k} k !} H_{\alpha+l-k}(f)(|y|) \Delta^{k} P(y)
\end{aligned}
$$


This implies in turn the following:

Corollary 5 (Periodicity relation for the Hankel transform). For any $\phi \in \mathcal{S}(\mathbb{R})$ with $\alpha$ and $l$ satisfying conditions of Corollary 2 above, the Hankel transform satisfies the following relation

$$
H_{\alpha+l}(\phi)(t)=2(\alpha+l-1) H_{\alpha+l-1}(\phi)(t)-H_{\alpha+l-2}\left(s^{2} \phi\right)(t) .
$$

\section{Conclusion}

In this paper we have demonstrated that differential identities for homogeneous polynomials like those implied by equations (3)-(5) can be effectively used for solving problems in harmonic analysis, which so far have been approached by means of integral identities of the type of HeckeBochner formula. In our opinion other possibilities of using that approach should certainly be further explored.

\section{Acknowledgements}

The results contained in this paper were presented at the conference Symmetry in Nonlinear Mathematical Physics in Kyiv, June 20-26, 2005 and also at the Seminar Sophus Lie in Nancy, June 10, 2005. We thank the organizers of those meetings for enabling us to present these results there. We are also obliged to the referees for remarks which, as we hope, enabled us to improve the presentation in the paper. In particular, the reference [4] was indicated by the referee.

[1] Andrews G.E., Askey R., Roy R., Special functions, Encyclopedia of Mathematics and its Applications, Vol. 71, Cambridge, Cambridge University Press, 1999.

[2] Bezubik A., Da̧browska A., Strasburger A., On the Fourier transform of $\mathbf{S O}(d)$-finite measures on the unit sphere, Arch. Math. (Basel), 2005, V.84, 470-480.

[3] Bezubik A., Dąbrowska A., Strasburger A., On spherical expansions of zonal functions on Euclidean spheres, submitted.

[4] zu Castell W., Filbir F., Radial Basis functions and corresponding zonal series expansions on the sphere, J. Approx. Theory, 2005, V.134, 65-79.

[5] Faraut J., Analyse harmonique et fonctions speciales, in Deux Cours d'Analyse Harmonique, Editors J. Faraut and K. Harzallah, Ecole d'Ètè d'Analyse Harmonique de Tunis, 1984, Basel, Birkhäuser Verlag, 1987.

[6] Gonzalez Vieli F.J., Inversion de Fourier ponctuelle des distributions à support compact, Arch. Math. (Basel), 2000, V.75, 290-298.

[7] Lucquiaud J.C., Generalization sous forme covariante des polynomes de Gegenbauer, J. Math. Pures Appl. (9), 1984, V.63, 265-282.

[8] Magnus W., Oberhettinger F., Soni R.P., Formulas and theorems for the special functions of mathematical physics, 3rd ed., Berlin, Springer-Verlag, 1966.

[9] Müller C., Analysis of spherical symmetries in Euclidean spaces, New York, Springer-Verlag, 1998.

[10] Stein E.M., Weiss G., Introduction to harmonic analysis on Euclidean spaces, Princeton, Princeton University Press, 1971.

[11] Strasburger A., A generalization of the Bochner identity, Exposition. Math., 1993, V.11, 153-157.

[12] Vilenkin N.J., Special functions and the theory of group representations, Moscow, Nauka, 1965 (in Russian).

[13] Wawrzyńczyk A., Group representations and special functions, Dordrecht - Warszawa, D. Reidel and PWN, 1984. 